\title{
Diagnostik und Therapie der zerebralen Venen- und Sinusthrombose
}

Christian Weimar

Auch wenn die zerebrale Venen-/Sinusthrombose (CVST) eine seltene zerebrovaskuläre Erkrankung darstellt, wird sie zunehmend häufiger diagnostiziert aufgrund erhöhter klinischer Aufmerksamkeit, besserer Bildgebungsmöglichkeiten sowie einer höheren Überlebensrate von Patienten mit prädisponierenden Erkrankungen. Dieser Beitrag zeigt, wie die Diagnose effektiv gestellt werden kann und welche Therapieoptionen in der Akutphase und Sekundärprophylaxe bestehen.

$\begin{array}{ll}\text { ABKÜRZUNGEN } \\ 95 \% \text { CI } & \text { 95\%-Konfidenzintervall } \\ \text { AV } & \text { arteriovenös } \\ \text { CTA } & \text { CT-Angiografie } \\ \text { CVST } & \text { zerebrale Venen-/Sinusthrombose } \\ \text { DOAK } & \text { direktes orales Antikoagulans } \\ \text { DSA } & \text { digitale Subtraktionsangiografie } \\ \text { FLAIR } & \text { Fluid-attenuated Inversion } \\ & \text { Recovery } \\ \text { HIT } & \text { heparininduzierte } \\ & \text { Thrombozytopenie } \\ \text { Ig } & \text { Immunglobulin } \\ \text { LMWH/NMH } & \text { low-molecular-weight Heparin / } \\ & \text { niedermolekulares Heparin } \\ \text { LP } & \text { Lumbalpunktion } \\ \text { PTT } & \text { partielle Thromboplastinzeit } \\ \text { SWI } & \text { Susceptibility weighted Imaging } \\ \text { UFH } & \text { unfraktioniertes Heparin } \\ \text { VTE } & \text { venöses thromboembolisches } \\ & \text { Ereignis } \\ & \end{array}$

\section{Epidemiologie}

Die Inzidenz einer zerebralen Venen-/Sinusthrombose (CVST) in zwei niederländischen Provinzen mit 3,1 Millionen Einwohnern betrug 1,32 pro 100000 Personenjahre (95\%-KI 1,06-1,61) bzw. 2,78 (95\%-KI 1,983,82) bei Frauen im Alter zwischen 31 und 50 Jahren [1]. Der häufigste Zeitpunkt liegt postpartal mit einer Inzidenz von etwa 10/100000 Entbindungen in Ländern der Ersten Welt, was bis zu 20\% aller Fälle von CVST entspricht [2].

\section{Ätiologie}

Die meisten prädisponierenden Faktoren einer CVST sind identisch mit den Risikofaktoren von peripheren venösen Thrombosen. Bei etwa 15\% der Patienten kann keine Ursache der CVST gefunden werden. Eine orale hormonelle Kontrazeption bleibt der einzige prädisponierende Faktor bei etwa $10 \%$ aller Patientinnen. Weitere Risikofaktoren sind in der folgenden tabellarischen Übersicht dargestellt.

Koagulopathien sind ein häufiger Befund bei CVST, insbesondere die Faktor-V-Leiden-Mutation, welche in 10-25\% aller Fälle (überwiegend heterozygot) vorliegt. Eine therapeutische Konsequenz ergibt sich daraus jedoch nur selten.

\begin{abstract}
Merke
Ein Screening auf Gerinnungsstörungen zur Prävention von erneuten venösen Thrombosen, zur Reduktion der Mortalität sowie zur Verbesserung des funktionellen Ergebnisses wird nicht generell empfohlen. Allenfalls kann, analog zum Vorgehen bei Beinvenenthrombose und Lungenembolie, bei Fehlen jedes Risikofaktors für die aktuelle Thrombose und bei positiver Familienanamnese für thromboembolische Ereignisse (erstgradige Verwandte) ein Thrombophiliescreening veranlasst werden, um eine hereditäre Thrombophilie neu zu entdecken.
\end{abstract}

\section{ÜBERSICHT}

Weitere Erkrankungen als Risikofaktoren für eine CVST

- Koagulopathien:

- Prothrombinmutation G 20210 A

- Antithrombin-III-Mangel

- Protein-C- und Protein-S-Mangel 
- Antiphospholipid-Antikörper-Syndrom

- Plasminogenmangel

- Hyperhomocysteinämie

- Dysfibrinogenämie

- disseminierte intravasale Koagulation

- Heparin-induzierte Thrombozytopenie Typ 2

- Malignome:

- Meningeom

- Karzinom

- Lymphom

- Karzinoid

- Leukämie

- hämatologische Erkrankungen:

- Polyzythämie

- Sichelzellkrankheit

- paroxysmale nächtliche Hämoglobinurie

- immunvermittelte hämolytische Erkrankungen

- Thrombozythämie

- Kollagenosen:

- systemischer Lupus erythematodes

- Sjögren-Syndrom

- Vaskulitiden:

- Morbus Behçet

- granulomatöse Angiitis

- Sarkoidose

- mechanische Ursachen:

- intrakranielle Hypotension

- Gehirnerschütterung

- neurochirurgische Eingriffe

- Verschlusshydrozephalus

- zentraler Venenkatheter

- durale arteriovenöse Malformation

- Strangulation

- Medikamente:

- Asparaginase

- andere Chemotherapeutika

- Steroide

- Erythropoietin

- Drogen

- Vitamin-A-Überdosierung

- metabolische Störungen:

- Diabetes mellitus

- Thyreotoxikose

- Urämie

- nephrotisches Syndrom

- gastrointestinale Erkrankungen:

- Leberzirrhose

- chronisch inflammatorische Darmerkrankungen

- kardiale Erkrankungen:

- Herzinsuffizienz

- Kardiomyopathie

- systemische Infektionen:

- bakterielle Sepsis

- Endokarditis
- Hepatitis

- Enzephalitis

- Masern

- Tuberkulose

- Typhus

- Malaria

- Aspergillose

- lokale Infektionen:

- Mastoiditis

- Otitis media

- Sinusitis

- Tonsillitis

- retropharyngeale Abszesse mit Thrombose der angrenzenden Jugularvene

- orale oder zerebrale Abszesse

- Meningitis

Malignome stellen zwar ebenfalls Risikofaktoren für eine CVST dar, ein systematisches Screening auf ein okkultes Malignom (einschließlich myeloproliferativer Erkrankungen) wird bei Patienten mit CVST dennoch nicht empfohlen. Eine CVST ohne Trigger kann jedoch Anlass sein, die alters- und geschlechtsspezifischen Tumorvorsorgeuntersuchungen zu aktualisieren.

\section{Pathophysiologie}

Zwei unabhängige Pathomechanismen sind zu unterscheiden:

- Thrombose der kortikalen Venen und

- Thrombose der großen Sinus.

Letztere sind wichtig für die Resorption von Liquor über die Pacchioni-Granulationen. Diese arachnoidalen Vorwölbungen in die zerebralen Sinus ermöglichen den Transport von Liquor aus dem Subarachnoidalraum in das Blut. Eine Thrombose der großen Sinus führt daher zu einer Liquorresorptionsstörung und damit zu intrakranieller Druckerhöhung. Ein Verschluss von zerebralen Venen verhindert dagegen die venöse Drainage von Blut aus dem Hirnparenchym und führt zur Erhöhung des venösen und kapillären Drucks mit Schädigung der Blut-HirnSchranke und resultierender Stauungsblutung ( $\triangleright$ Abb. 1).

\section{Klinik}

\section{FALLBEISPIEL}

Der 30-jährige, bis dahin gesunde Patient stellte sich aufgrund von starken Kopfschmerzen vor, welche im Anschluss an einen fieberhaften Infekt vor 4 Wochen begonnen hatten. Seit einigen Tagen hätten die Kopfschmerzen deutlich zugenommen und seien nun rechts betont ohne vegetative Begleitsymptome. 
In der klinischen Untersuchung ergab sich ein regelrechter Befund. Eine Stauungspapille konnte auch augenärztlich nicht nachgewiesen werden.

Eine CVST kann oft klinisch stumm verlaufen, da eine Vielzahl von venösen Drainagewegen und Flussvarianten existiert. Kopfschmerz ist in mehr als $70 \%$ das erste Symptom und bleibt auch das einzige Symptom in etwa $16 \%$ der Fälle [3]. Es können zusätzlich Übelkeit/Erbrechen, epileptische Anfälle, Bewusstseinsstörungen oder Psychosen und andere fokal neurologische Ausfälle auftreten [4]. Ein Papillenödem mit oder ohne begleitende Visusstörung tritt in etwa $40 \%$ auf, vor allem bei Patienten mit einem chronischen Verlauf und verzögerter Diagnosestellung. Fokale oder generalisierte epileptische Anfälle werden bei 30-40\% der Patienten beobachtet. Weitere, seltenere klinische Symptome sind

- ein Donnerschlag-Kopfschmerz,

- Hirnnervenausfälle,

- transiente ischämische Attacken,

- Migräne mit Aura,

- psychiatrische Störungen und

- Tinnitus.

Die Klinik der neurologischen Ausfälle bzw. epileptischen Anfälle wird zum Teil durch die Lokalisation der Throm-

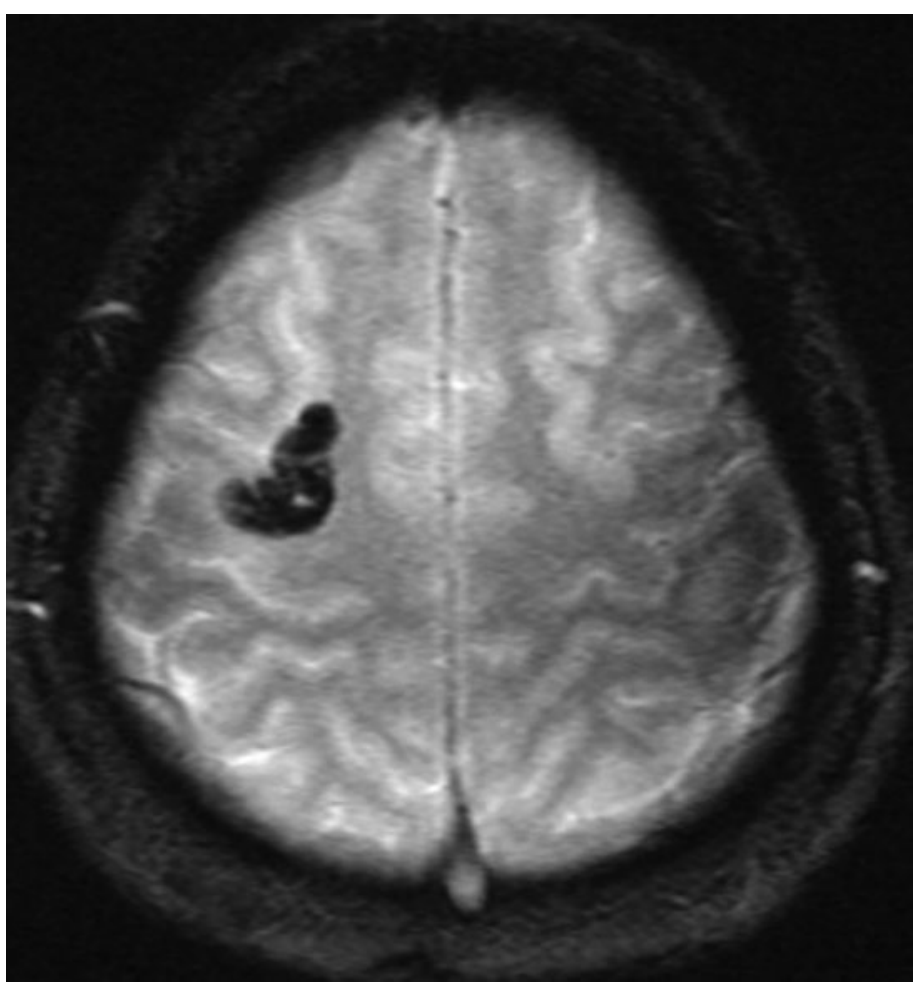

-Abb. 1 T2* -gewichtete axiale MRT einer 30-jährigen Patientin mit kortikaler Blutung aufgrund einer Thrombose einer rechts frontalen kortikalen Vene. bose und der hierdurch verursachten Läsionen bestimmt. Die häufigste Thrombose im Sinus sagittalis superior (60\% aller Fälle) verursacht Kopfschmerzen, Papillenödeme, epileptische Anfälle, motorische Ausfälle und Bewusstseinsstörung. Eine Thrombose des Sinus cavernosus ist assoziiert mit Augenmuskellähmungen und weiteren okulären Symptomen wie Chemose, Proptose, Papillenödem. Patienten mit isolierter Thrombose der V. Labbé zeigen häufig Hirndrucksymptome oder eine Aphasie bei Thrombose auf der Seite der sprachrelevanten Hemisphäre.

Eine Thrombose der tiefen Hirnvenen verursacht oft Bewusstseinsstörungen, motorische Ausfälle oder eine Aphasie. Eine bilaterale venöse Thrombose im Thalamus führt ebenfalls zu Bewusstseinsstörungen als Hauptsymptom oder auch nur leichten vorübergehenden kognitiven Einschränkungen bis hin zu einem schweren amnestischen Syndrom.

\section{Bildgebung}

Merke

Bei klinischem Verdacht auf eine CVST muss unverzüglich eine bildgebende Diagnostik erfolgen.

\section{Computertomografie}

Der computertomografische Nachweis einer CVST erfolgt am besten mittels dynamischer, kontrastmittelangehobener CT-Angiografie (CTA). Diese sollte mit einer Schichtdicke von 1-1,5 mm in der venösen Phase des Kontrastmittelbolus durchgeführt werden. Die Analyse der Quellenbilder kann durch multiplanare Rekonstruktionen ergänzt werden.

\section{Magnetresonanztomografie}

Aufgrund der fehlenden Strahlenbelastung wird die Magnetresonanztomografie (MRT) vor allem bei jüngeren Patienten sowie in der Schwangerschaft eingesetzt. Eine native MRT schließt allerdings eine CVST nicht aus. Das Signal von thrombosiertem Blut ist abhängig vom Alter des Thrombus. Axiale und sagittale T1- und T2-Bilder zeigen keine Signalauslöschung im thrombosierten Sinus, sondern im Idealfall sogar eine deutliche Signalanhebung durch den Methämoglobingehalt des Thrombus.

Der direkte Thrombusnachweis gelingt mit T1- oder T2* gewichteten bzw. Susceptibility weighted Imaging-(SWI-) Aufnahmen in Abhängigkeit von Thrombuslokalisation und -alter zumeist sehr sensitiv. Hiermit lassen sich neben den verschlossenen Sinus auch einzelne thrombosierte Venen als hypointense Strukturen darstellen. Diese müssen allerdings gegenüber lokalen subpialen oder subarachnoidalen Blutungen abgegrenzt werden, die sich in der T2*-Wichtung ebenfalls hypointens darstellen. Alternativ kann auch eine MP-RAGE Sequenz benutzt werden, in der sich die 
thrombosierte kortikale Vene hyperintens darstellt. Als Ausdruck einer isolierten Brückenvenenthrombose findet sich manchmal eine umschriebene sulkale Subarachnoidalblutung ( $\triangleright$ Abb. 2).

Nach Kontrastmittelgabe ist die Kontrastmittelaussparung im thrombosierten Sinus ähnlich wie im Computertomogramm erkennbar ( $\triangleright$ Abb. 3 ).

Nicht selten führen jedoch Anlagevarianten der Sinus sowie intrasinusoidale Strukturen (Pacchioni-Granulationen, Septen etc.) zu falsch positiven Befunden mit konsekutiver Antikoagulation. So muss z. B. eine Hypoplasie des Sinus transversus von einer Thrombose unterschieden werden. Hierfür wurde bei fehlendem Flusssignal das Sigmoid Notch Sign als Hinweis für eine Hypo- oder Aplasie des Sinus transversus beschrieben ( $\triangleright$ Abb. 4) [5].

Intrazerebrale Blutungen finden sich bei 30-40\% aller Patienten, hingegen sind subdurale oder subarachnoidale Blutungen deutlich seltener [4]. Eine Thrombose der $\mathrm{V}$. Labbé kann eine große temporale Blutung verursachen, welche nicht mit einer Aneurysmablutung verwechselt werden sollte ( $\triangleright$ Abb. 5 ).

Die Mehrzahl der Blutungen treten als venöse hämorrhagische Infarkte in Ödemzonen auf, welche meist noch nicht infarziert und reversibel sind. Kleine juxtakortikale Blutungen in der Grenzzone zwischen oberflächlichem und tiefem Venensystemen ohne oder mit nur geringem Begleitödem sind typisch für Thrombosen des Sinus sagittalis superior ( $\triangleright$ Abb. 6 ). Insbesondere ein isoliertes Hirnödem der Basalganglien und Thalami bei Thrombose der inneren Hirnvenen kann besser mittels MRT dargestellt werden ( Abb. 7).

\section{FAZIT}

Die Computertomografie (CT) und Magnetresonanztomografie (MRT), jeweils mit venöser Angiografie, sind als gleichwertig bei der Diagnostik der Sinusthrombosen anzusehen. Bei kortikalen Venenthrombosen sowie Thrombosen tiefer Hirnvenen ist die MRT der CT überlegen. Aufgrund der fehlenden Strahlenbelastung soll die MRT bevorzugt bei jüngeren Patienten sowie in der Schwangerschaft eingesetzt werden.

Die digitale Subtraktionsangiografie DSA ist nur noch selten indiziert zum Nachweis von kortikalen Thrombosen. Ansonsten spielt sie heute bei der Diagnostik der CVST praktisch keine Rolle mehr.

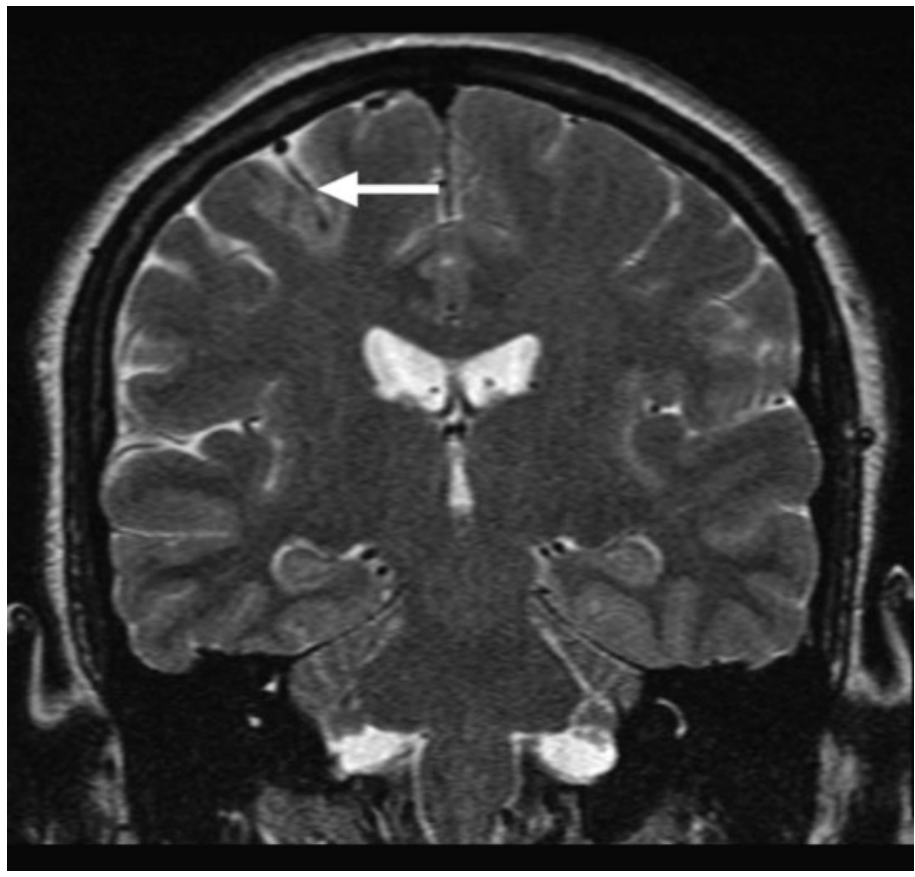

- Abb. 2 T2-gewichtete koronare MRT einer 24-jährigen Patientin mit subarachnoidaler Blutung in einem Sulkus aufgrund einer Thrombose einer kortikalen Vene.

\section{D-Dimer-Testung}

\section{FALLBEISPIEL}

Laborchemisch waren die D-Dimere im Serum leicht erhöht.

In der primär durchgeführten MRT mit venöser Angiografie wurde eine ausgedehnte Sinusthrombose mit Beteiligung des Sinus sagittalis superior, Sinus rectus, Sinus transversus, und Sinus sigmoideus rechts nachgewiesen. Der übrige zerebrale Befund war unauffällig. Die Thrombophiliediagnostik wies eine leicht verminderte Protein-S-Aktivität (38\%) nach. Die Bestimmung der Anti-Phospholipid-Antikörper zeigte niedertitrige lgG-Antikörper gegen $\beta_{2}$-Glykoprotein.

Für Patienten mit isolierten Kopfschmerzen, d.h. mit einer vergleichsweise niedrigen Vortestwahrscheinlichkeit für eine CVST, wurde kürzlich in einer Metaanalyse zwar gezeigt, dass D-Dimere einen hohen negativen prädiktiven Wert haben, d.h. eine CVST bei negativen D-Dimeren mit einer Sicherheit von 99,8\% ausgeschlossen werden kann. In anderen Studien aber lag das Risiko vergleichsweise hoch, eine CVST bei negativem D-Dimer-Test zu übersehen, insbesondere bei Patienten mit isolierten Kopfschmerzen oder seit mehr als einer Woche bestehender Beschwerdesymptomatik. 


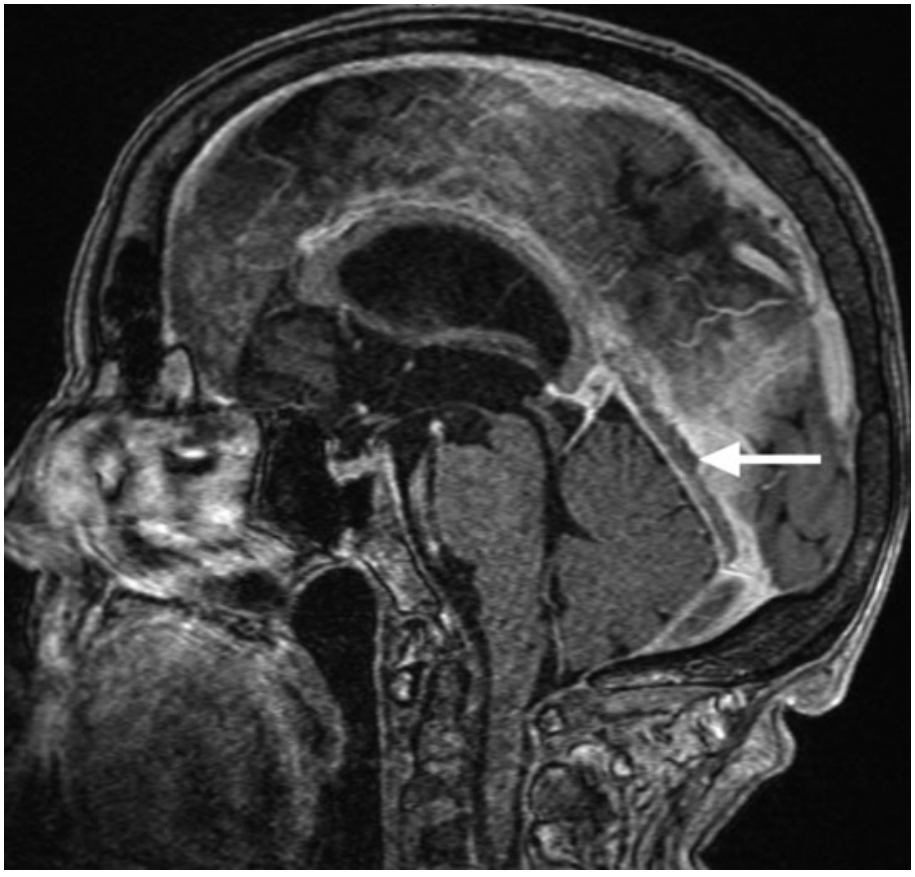

-Abb. 3 Venöse MR-Angiografie bei einer 76-jährigen Patientin mit Kontrastmittelaussparung aufgrund einer Thrombose des Sinus rectus.

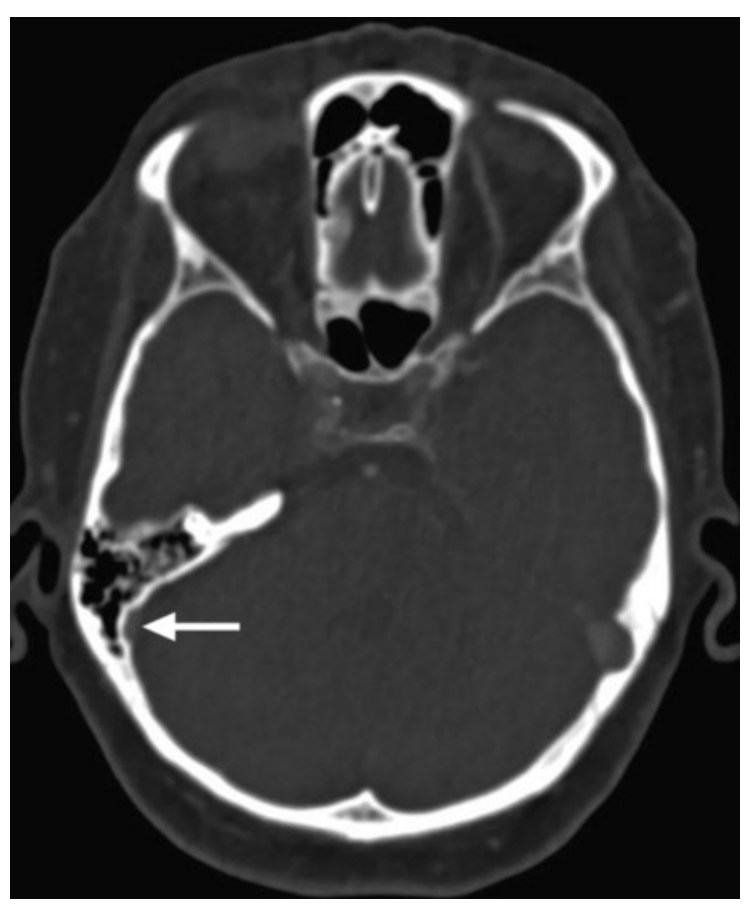

Abb.4 Asymmetrie der Knochenaussparung um den Sinus sigmoideus als Hinweis für eine Hypoplasie rechts.

Anders als für die tiefe Beinvenenthrombose oder die Lungenembolie stehen bei Verdacht auf CVST keine validierten Skalen zur Verfügung, die es erlauben, anhand klinischer Merkmale oder Risikofaktoren eine Vortestwahrscheinlichkeit zu ermitteln. Dies wäre für eine systematische Verwendung des nachfolgenden DDimer-Tests aber erforderlich.

\section{PRAXIS}

\section{Stellenwert}

Die Entscheidung für oder gegen eine zerebrale Bildgebung sollte nicht vom Ergebnis eines D-DimerTests abhängig gemacht werden. Diesem kommt allenfalls eine unterstützende Funktion mit unklarem Stellenwert im diagnostischen Ablauf zu .

\section{Therapeutisches Vorgehen in der Akuttherapie}

\section{Allgemeine Maßnahmen}

Wie auch beim Schlaganfall sollte die Akutbehandlung unter Monitorbedingungen auf einer neurologischen Stroke Unit erfolgen, um eine klinische Verschlechterung oder Komplikationen frühzeitig zu erkennen und behandeln zu können. Spätestens bei Auftreten von Hirndruckzeichen ist eine Verlegung in ein Zentrum mit (interventioneller) Neuroradiologie und Neurochirurgie zu empfehlen.

\section{Antikoagulation}

Analog zur Behandlung extrazerebraler venöser Thrombosen werden Patienten mit einer CVST in der Akutphase mit Heparin antikoaguliert. Diese Behandlung erfolgt mit dem Ziel, eine Propagation des Thrombus bzw. den erneuten thrombotischen Verschluss von bereits durch die körpereigene Fibrinolyse wieder geöffneten Gefäßabschnitten zu verhindern.

\section{Merke \\ Patienten mit CVST sollen in der akuten Phase mit Heparin in einer therapeutischen Dosis behandelt wer- den, unabhängig davon, ob bereits eine intrakranielle Blutung vorliegt oder nicht.}

Die Evidenz hierzu beruht auf einer Metaanalyse aus 2 randomisierten und placebokontrollierten klinischen Studien mit insgesamt 79 Patienten. Hier zeigte sich, dass eine Antikoagulation mit unfraktioniertem (UFH) oder niedermolekularem Heparin (NMH) das Risiko für ein schlechtes klinisches Ergebnis um 54\% (Tod oder Pflegebedürftigkeit, 95\%-KI 0,16-1,31) und das Risiko zu sterben um $67 \%$ reduzierte $(95 \%-K I$ 0,08-1,21) [6]. Allerdings war diese Risikoreduktion statistisch nicht signifikant, was höchstwahrscheinlich an der zu geringen Patientenzahl lag. Beide Studien konnten jedoch die Sicherheit einer therapeutischen Antikoagulation auch bei Patienten mit einer vorbestehenden intrakaniellen Blutung zeigen.

Da die Behandlung mit NMH keinen intravenösen Zugang und keine regelmäßigen Laborkontrollen 


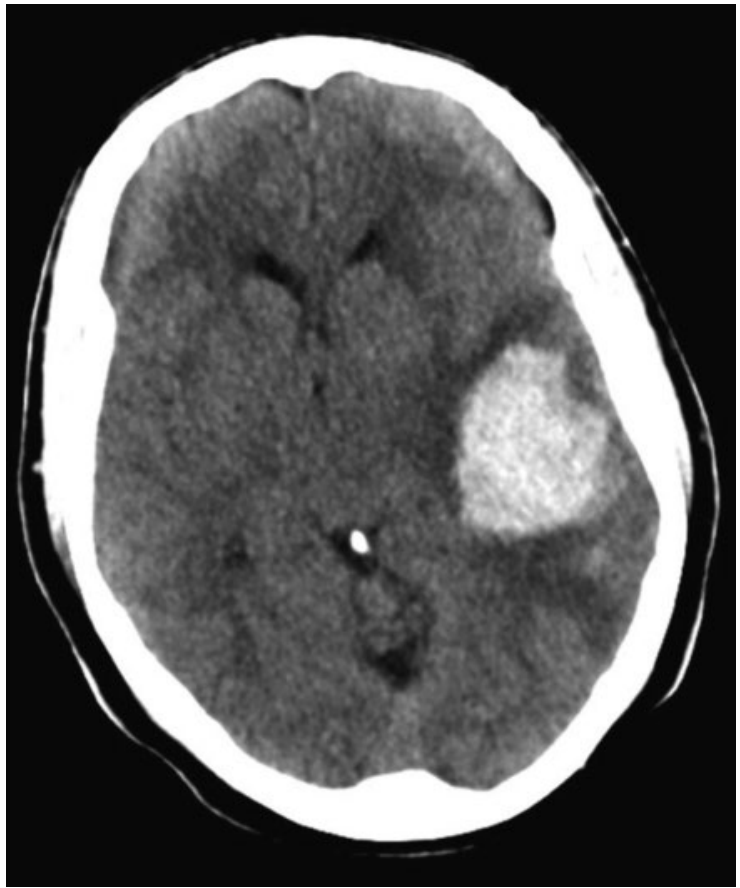

-Abb.5 Temporale Blutung im CT einer 59-jährigen Patientin mit Thrombose der V. Labbé.

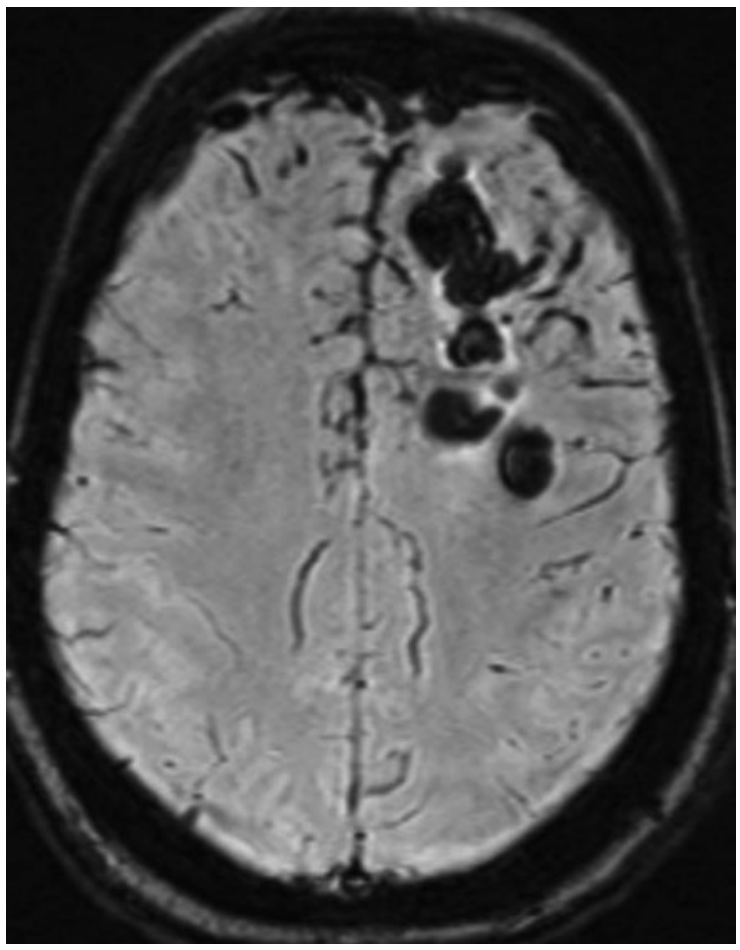

-Abb. 6 Suszeptibilitätsgewichtete axiale MRT mit Nachweis von juxtakortikalen Einblutungen in der Grenzzone zwischen oberflächlicher und tiefer venöser Drainage mit umgebendem Ödem bei einer 26-jährigen Patientin mit Thrombose des Sinus sagittalis superior.

erfordert, wird dieses Vorgehen in der praktischen Anwendung oft bevorzugt. Für extrazerebrale venöse
Thrombosen und Lungenembolien zeigte sich in einer Metaanalyse, dass die Behandlung mit $\mathrm{NMH}$ mit einer signifikant niedrigeren Letalität (OR 0,62; 95\%-KI $0,46-0,84)$ und erheblich weniger schweren Blutungskomplikationen (OR 0,5; 95\%-KI 0,29-0,85) im Vergleich zu einer Behandlung mit UFH verbunden war. Die europäische CVST-Leitlinie empfiehlt ebenso wie die AWMF-S2-Therapieleitlinien zur Diagnostik und Therapie der peripheren Venenthrombose und der Lungenembolie die Behandlung mit gewichtsadaptierten $\mathrm{NMH}$ [7], [8].

\section{Merke}

Patienten mit einer akuten CVST können vorzugsweise mit niedermolekulares Heparin statt unfraktioniertem Heparin behandelt werden.

$\mathrm{NMH}$ führen wesentlich seltener als unfraktionierte Heparine zu einer heparininduzierten Thrombozytopenie (HIT) Typ II. Klinische Probleme mit Thrombozytenabfall und konsekutiven neuen Gefäßverschlüssen sind mit beiden Heparinarten nicht vor dem 5. und selten nach dem 14.Tag zu erwarten, sofern keine Vorbehandlung erfolgte. Kontrollen der Thrombozytenzahl sollten deshalb bei einer Behandlungsdauer von mehr als 5 Tagen für 2 Wochen vorgenommen werden [8].

Einen möglichen Vorteil hat UFH bei intensivpflichtigen Patienten mit eventuell kurzfristig erforderlicher operativer Intervention, da hier nach Beendigung der intravenösen Heparintherapie innerhalb von 1-2 Stunden eine Normalisierung der Gerinnung eintritt. Auch Patienten, die eine Kontraindikation für den Einsatz von $\mathrm{NMH}$ aufweisen (schwere Niereninsuffizienz), sollten mit UFH behandelt werden.

Derzeit ist die Datenlage nicht ausreichend, um den Einsatz von direkten oralen Antikoagulanzien (Faktor-XaInhibitoren oder Thrombininhibitoren) für die Behandlung von CVST-Patienten in der akuten Phase zu empfehlen.

\section{Schwangerschaft und Geburt}

Zur Behandlung einer CVST während einer Schwangerschaft kann peri- und postpartal sowohl NMH als UFH eingesetzt werden. Diese Antikoagulation sollte rund um die Geburt (Spontangeburt, Sectio, Anlage Periduralanästhesie etc.) durch ein Expertenteam gesteuert und überwacht werden. Eine Antikoagulation in der Akutphase wird ebenfalls bei Kindern empfohlen [9].

\section{Infektiöse Thrombose}

Septische oder infektiöse Thrombosen, die fast ausschließlich als Komplikation einer lokalen Infektion (z. B. einer Sinusitis oder Mastoiditis) auftreten, werden heute in Industrieländern nur noch sehr selten beobachtet. Sie werden antibiotisch gemäß Keimnachweis und 
Resistogramm behandelt und es erfolgteine operative Sanierung des Infektfokus. Eine Antikoagulation erfolgt ansonsten wie bei nicht septischen Thrombosen.

\section{Endovaskuläre Behandlung}

Endovaskuläre Verfahren stellen eine Therapieeskalation dar und beruhen auf einer langsamen Fibrinolysebehandlung des Thrombus von arterieller Seite über einen Mikrokatheter, ggf. in Kombination mit transvenöser mechanischer Rekanalisation, z.B. mittels eines Absaugkatheters.

Die erste und einzige randomisierte Studie (TO-ACT), bei der Patienten mit einem hohen Risiko für ein schlechtes klinisches Ergebnis mit einer endovaskulären Thrombolyse versus therapeutischer Antikoagulation behandelt werden, wurde vorzeitig beendet und zeigte keinen Vorteil der invasiven Behandlung [10]. Der Anteil von Patienten mit einem sehr guten Therapieergebnis (mRS 0-1) war in beiden Gruppen nahezu identisch (67\% versus 68 $\%)$, allerdings verstarben in der endovaskulär behandelten Gruppe mehr Patienten (4 versus 1). Patienten mit einer CVST, die ein niedriges Risiko für ein schlechtes klinisches Ergebnis aufweisen, sind daher keine geeigneten Kandidaten für eine Thrombolyse. Auch Patienten mit großen raumfordernden hämorrhagischen Infarkten profitieren wahrscheinlich nicht von einer thrombolytischen Therapie, da eine Größenzunahme der Blutung den Prozess der drohenden Einklemmung beschleunigen kann. Ein systematischer Review der vorhandenen Evidenz zur Thrombolyse zeigte eine Inzidenz von schweren

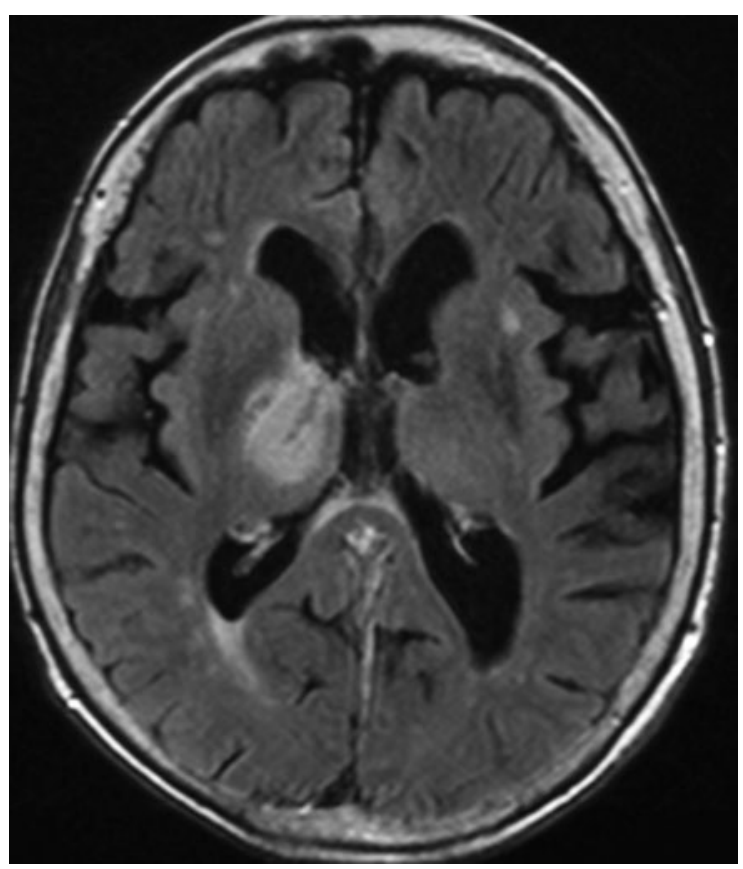

Abb. 7: Axiale FLAIR- (Fluid-attenuated Inversion Recovery) gewichtete MRT eines 77-jährigen Patienten mit Thrombose der tiefen Hirnvenen und Ödem im rechten Thalamus.
Blutungskomplikationen von 9,8\% (95\%-KI 5,3-15,6\%) [11]. Symptomatische intrakranielle Blutungen traten bei 7,6\% auf, und die Mortalität lag bei 9,2\%. Die postinterventionellen Rekanalisationsraten (partiell oder komplett mit mechanischer Thrombektomie) wurden mit 95\% angegeben.

\section{Merke}

Zum jetzigen Zeitpunkt sollte eine interventionelle Behandlung nur in Einzelfällen und in spezialisierten Zentren erfolgen, wenn es unter einer suffizienten Antikoagulation (und nach Ausschluss anderer Komplikationen wie beispielsweise epileptischen Anfällen oder Infektionen) zu einer progredienten Verschlechterung kommt.

\section{Hirndruckbehandlung}

Obwohl bei bis zu 50\% aller Patienten mit zerebraler Venen-/Sinusthrombose in der Bildgebung ein Hirnödem nachweisbar ist, sind spezifische hirndrucksenkende Maßnahmen nur in einer geringen Anzahl der Fälle notwendig.

Die beste Hirndruckbehandlung ist eine ausreichende Antikoagulation, da hier der venöse Abfluss verbessert wird, was zu einer Reduktion des intrakraniellen Druckes führt. Eine Volumenrestriktion zur Hirndrucksenkung ist zu vermeiden.

\section{Kranielle Dekompression}

Zur operativen Dekompression liegen lediglich teils kontrollierte Fallserien sowie Reviews vor. Auch wenn die Fallzahlen insgesamt klein sind, deuten die Ergebnisse übereinstimmend darauf hin, dass sich bei drohender Einklemmung mittels operativer Dekompression Todesfälle verhindern lassen, ohne gleichzeitig zu einer Zunahme des Anteils schwerstbehinderter Patienten zu führen. Zudem werden einige Fälle berichtet, bei denen trotz fortgeschrittener klinischer Herniationszeichen ein funktionell gutes Ergebnis resultierte.

\footnotetext{
Merke

Trotz der geringen Evidenz wird eine operative Dekompression bei Patienten mit CVST, Läsionen des Parenchyms (Stauungsödem und / oder Hämorrhagie) und drohender Einklemmung zur Verhinderung von Todesfällen empfohlen.
}

\section{Kortikosteroide}

Steroide sollen aufgrund ihrer prothrombotischen Wirkungen und ihrer fehlenden Wirksamkeit nicht gegeben werden. Ausnahmen sind Patienten mit CVST aufgrund autoimmunentzündlicher Erkrankungen wie z. B. Morbus Behçet oder systemischer Lupus erythematodes.

\section{Liquordrainage bei Hydrozephalus}

Da kein Druckgradient zwischen den Ventrikeln und der Hirnoberfläche entsteht, ist ein Hydrozephalus bei CVST 
eher selten und meist die Folge einer mechanischen Verlegung, z.B. bei bilateralem Thalamusödem bzw. einer Schwellung in der hinteren Schädelgrube oder Komplikation einer ventrikulären Einblutung. In solchen Einzelfällen kann die Anlage einer Liquordrainage erforderlich sein.

\section{Lumbalpunktion}

Eine diagnostische oder therapeutische Lumbalpunktion (LP) bei akuter CVST hatte im Rahmen der International Study on Cerebral Vein and Dural Sinus Thrombosis (ICVST) keinen Einfluss auf das akute oder mittelfristige klinische Ergebnis [7]. In der Postakutphase und bei Patienten mit klinischen Symptomen einer intrakraniellen Druckerhöhung, die nicht auf eine Hämorrhagie oder einen Stauungsinfarkt zurückzuführen sind, stellt die therapeutische LP vermutlich eine sichere Methode mit potenziell günstigem Effekt auf Kopfschmerz und bei drohendem Visusverlust dar. Hierzu können ggf. wiederholte Punktionen erforderlich sein.

\section{Orale Antikoagulation}

\section{FALLBEISPIEL}

Nach Abnahme der Labordiagnostik wurde eine PTTwirksame Heparinisierung begonnen, welche nach 3 Tagen auf Phenprocoumon umgestellt wurde. Eine MRT-Kontrolle 6Monate später zeigte residuelle Thromben mit Teilrekanalisierung der Sinus. Eine Vorstellung in einer Gerinnungsambulanz ergab neben einer erhöhten Faktor-VIII-Aktivität eine Erniedrigung von Protein $\mathrm{C}$ und $\mathrm{S}$, was unter der Behandlung mit Vitamin-K-Antagonisten nicht aussagekräftig war.

Prospektive Daten zur optimalen Dauer einer oralen Antikoagulation liegen nicht vor. Eine retrospektive Studie mit 706 Patienten und einer medianen Nachbeobachtungszeit von 40 Monaten zeigte ein CVST-Rezidiv bei $4,4 \%$ und eine extrazerebrale venöse Thrombose bei 6,5\% der Patienten mit einer Gesamtinzidenz für Rezidive von 23,6 auf 1000 Patientenjahre (95\%-KI $17,8-28,7)$ und 35,1 pro 1000 Patientenjahre (95\%-KI 27,7-44,4) nach Beendigung der oralen Antikoagulation [12]. Eine venöse Thromboembolie in der Vorgeschichte war der einzige signifikante Prädiktor für ein erneutes Rezidiv in der multivariaten Analyse.

\section{PRAXIS}

Prinzip

Eine orale Antikoagulation mit einem Vitamin-KAntagonisten wird für 3-12 Monate empfohlen zur Prävention einer Rezidiv-CVST und extrazerebraler venöser Thrombosen .
In Ermangelung klarer Evidenz empfiehlt auch die aktuelle europäische Leitlinie eine langfristige Antikoagulation unter regelmäßiger Nutzen-Risiko-Bewertung nur bei einer zugrunde liegenden schwerwiegenden Thrombophilie. Analog zu Patienten mit rezidivierenden extrazerebralen Thrombosen oder einer schwerwiegenden Thrombophilie (Antiphospholipidsyndrom, homozygoter Prothrombin-G20210A-Mutation, homozygoter FaktorV-Leiden-Mutation, Protein-C-, -S- oder Antithrombinmangel oder bei kombinierten Thrombophilien) kann unter regelmäßiger Nutzen-Risiko-Bewertung eine Langzeitantikoagulation durchgeführt werden.

Direkte orale Antikoagulanzien (DOAK) haben in großen Studien bei Patienten mit nicht rheumatischem Vorhofflimmern oder tiefen Beinvenenthrombosen und Lungenembolien im Vergleich zu einer Behandlung mit einem Vitamin-K-Antagonisten bei vergleichbarer Effektivität ein deutlich besseres Sicherheitsprofil aufgewiesen. Thrombinsowie Faktor Xa-Antagonisten werden daher in der klinischen Praxis zunehmend als Alternative zu Vitamin K-Antagonisten auch in der Postakutphase nach CVST eingesetzt. Daten zum Einsatz von DOAK in der Post-Akutphase nach CVST liegen zwischenzeitlich aus der RESPECT-CVT Studie vor, welche randomisiert Dabigatran $2 \mathrm{x}$ $150 \mathrm{mg}$ mit Warfarin (Ziel INR 2-3) verglich. Auch wenn diese Studie mit insgesamt 120 Patienten nicht für einen statistischen Vergleich gepowert war, zeigten sich im Behandlungszeitraum ab 5-15 Tage nach Erstdiagnose während 24-wöchiger Behandlung mit Dabigatran versus Warfarin ähnlich geringe Raten von schweren Blutungsereignissen oder erneuten venösen Thrombosen (1.7 versus $3.3 \%$ ) sowie hohe Rekanalisationsraten (60 versus $67 \%$ ).

\author{
Merke \\ Trotz weiterhin begrenzter Evidenz liegen jetzt pro- \\ spektive und kontrollierte Studiendaten vor, auf die \\ beim Einsatz von DOAKs bei Patienten mit CVST nach \\ der akuten Phase verwiesen werden kann. \\ Cave \\ Derzeit ist die Datenlage nicht ausreichend, um DOAKs \\ (Faktor-Xa-Inhibitoren oder Thrombin-Inhibitoren) für \\ die Behandlung von CVST-Patienten in der akuten \\ Phase einzusetzen.
}

\section{Prognose}

\section{FALLBEISPIEL}

Nach erneutem Nachweis von Restthromben in einer MR-Angiografie nach einem Jahr gab der Patient ein neues pulssynchrones Ohrgeräusch auf der rechten Seite an. Eine ergänzende MRT mit TWIST-(zeitaufgelöster Kontrastmittel-)Angiografie zeigte eine durale AV-Fistel Cognard Typ Ila am Sinus sigmoideus rechts, 


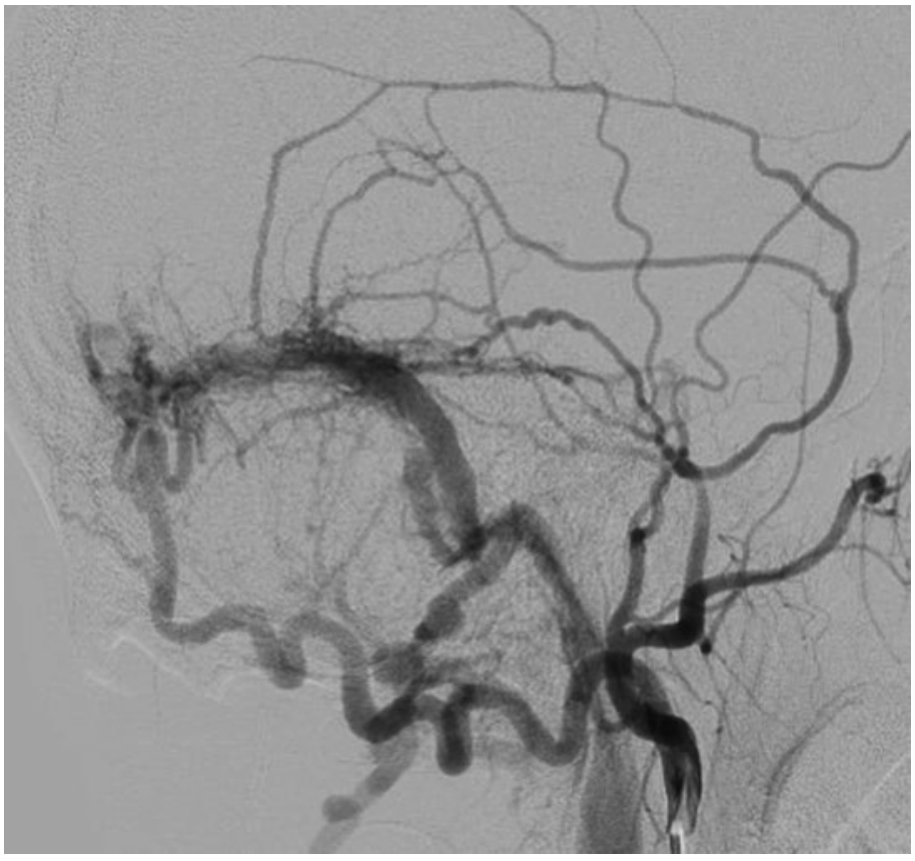

Abb.8 MR-Angiografie mit Nachweis einer duralen AV-Fistel rechts okzipital.

die nach diagnostischer Angiografie ( $\triangleright$ Abb. 8) elektiv embolisiert wurde. Die Antikoagulation wurde zuvor beendet und eine prophylaktische Gabe von niedermolekularem Heparin lediglich in Risikosituationen empfohlen.

Aufgrund der zunehmend besseren Diagnosemöglichkeit wird eine CVST auch bei oligosymptomatischen Patienten, z.B. mit isoliertem Kopfschmerz oder Papillenödem, zunehmend häufiger diagnostiziert. Hierdurch sowie durch Antikoagulation in der Akutphase sind Mortalitäts- und Morbiditätsraten von ursprünglich $50 \%$ vor 30 Jahren auf $15 \%$ in aktuellen Fallserien zurückgegangen [14]. Nichtsdestotrotz kommt es noch bei etwa $1 / 3$ aller Patienten zu kognitiven Einschränkungen und bei der Hälfte zu persistierenden Kopfschmerzen, Anpassungs- oder Angststörungen [15], [16].

Epileptische Anfälle treten bei 40-50\% der Patienten mit CVST auf und können zu einer abrupten und anhaltenden klinischen Verschlechterung führen [17],[18]. Insbesondere Patienten mit kortikalen Venenthrombosen, motorischen und/oder sensiblen Defiziten und hämorrhagischen Infarkten sind bezüglich des Auftretens von frühen epileptischen Anfällen gefährdet [19]. In einer prospektiven Observationsstudie konnte eine frühe antiepileptische Therapie bei Patienten mit CVST und supratentoriellen Läsionen nach einem epileptischen Anfall als klinisches Erstsymptom das Risiko eines weiteren epileptischen Anfalles deutlich verringern [18].

Es existiert keine Evidenz für eine routinemäßige vorbeugende antiepileptische Behandlung bei CVST, diese kann allenfalls bei Patienten mit hohem Risiko von Anfällen als Einzelfallentscheidung erwogen werden. Da das Risiko einer persistierenden Epilepsie als gering angenommen wird, wird eine Behandlungsdauer von 3-6 Monaten empfohlen, bei Patienten mit hohem Risiko (kortikalen Venenthrombosen, motorischen und/oder sensiblen Defiziten und hämorrhagischen Infarkten) auch bis zu 12 Monate.

Merke

Eine medikamentöse antiepileptische Behandlung sollte nur bei Patienten mit CVST nach stattgehabtem epileptischem Anfall gegeben werden, um erneute epileptische Anfälle zu vermeiden.

\section{Rezidivprophylaxe in Risikosituationen}

Die Rezidivrate für venöse thromboembolische Ereignisse (VTE) nach einer CVST lag in einer multizentrischen retrospektiven Studie bei 706 Patienten mit Erstmanifestation bei 2,36 pro 100 Patientenjahre (95\%-KI 1,78$2,87)$. Dieses Thromboserisiko persistierte auch in einer Langzeituntersuchung an 187 Patienten und war nach im Mittel 6 Jahren noch nachweisbar [20].

Für Erwachsene mit vorausgehender CVST gilt die entsprechende Empfehlung der aktuellen AWMF-S3-Leitlinien zur Prophylaxe der venösen Thromboembolie für die Gruppe mit hohem venösem Thromboembolierisiko, d.h. in Risikosituationen sollte eine medikamentöse Prophylaxe mit einem Antikoagulans durchgeführt werden [21].

Auch Kinder und Jugendliche, die bereits eine CVST erlitten haben, sollten in Risikosituationen, wie z. B. bei einer Immobilisierung > 4 Tage, Erkrankungen aus dem rheumatischen und onkologischen Formenkreis, erneuter Exposition von E. coli, Asparaginase und Steroiden, Anlage zentraler Venenkatheter, Flugreisen $>4$ Stunden Dauer, eine Thromboembolieprophylaxe mit gewichtsadaptiertem, niedermolekularem Heparin erhalten [22].

Die Einnahme von kombinierten oralen Kontrazeptiva erhöht das relative Risiko, erneut eine CVST zu erleiden, um den Faktor 7,6, wobei diese Assoziation für die Entstehung eines CVST-Rezidivs stärker zu bewerten ist als für ein erneutes Auftreten eines VTE [7]. Dieses erhöhte Rezidivrisiko betrifft auch neuere östrogenhaltige Präparate, wobei eine separate Analyse zur Anwendung von Progesteron-Monopräparaten aussteht. Aus diesen Gründen sollten Frauen mit vorausgehender CVST über die Möglichkeit eines Rezidivs unter erneuter Einnahme einer hormonellen Kontrazeption informiert werden und nach Möglichkeit auf eine Weiterführung oder den Neubeginn einer oralen Kontrazeption verzichten. 
Im Vergleich zu Gesunden haben Frauen nach einer CVST in einer Schwangerschaft ein niedriges absolutes Risiko von 9/1000, erneut eine CVST zu erleiden [23]. Das Risiko des Auftretens eines anderweitigen VTE in der Schwangerschaft wurde in diesem systematischen Review mit 27/1000 Schwangerschaften angegeben. Die gleiche Arbeit kommt zu dem Schluss, dass eine frühere CVST keinen Risikofaktor für das Auftreten von Spontanaborten darstellt. Frauen mit Z.n. CVST sollten daher aufgeklärt werden

- über das niedrige Rezidivrisiko, erneut an einer CVST in einer Folgeschwangerschaft zu erkranken, und

- über die fehlende Assoziation zum Auftreten von Spontanaborten.

Der Nutzen einer Thromboseprophylaxe während der Schwangerschaft bei Frauen mit früherer CVST konnte bisher in dem systematischen Review aus 13 Beobachtungsstudien nicht klar beantwortet werden [23] .Daher werden die Empfehlungen in Analogie zum Vorgehen bei früheren extrazerebralen venösen Thrombosen (VTE) bei Frauen abgeleitet, wobei sich ein positiver Trend zugunsten einer Thromboseprophylaxe zeigt [24].

\section{Merke}

Frauen mit früherer CVST ohne Kontraindikation für eine prophylaktische oder therapeutische LMWHApplikation sollten in einer erneuten Schwangerschaft und im Wochenbett eine subkutan zu verabreichende LMWH-Prophylaxe erhalten.

\section{KERNAUSSAGEN}

- Die Computertomografie (CT) und Magnetresonanztomografie (MRT), jeweils mit venöser Angiografie, sind als gleichwertig bei der Diagnostik der Sinusthrombosen anzusehen. Bei kortikalen Venenthrombosen ist die MRT der CT überlegen. Aufgrund der fehlenden Strahlenbelastung soll die MRT bevorzugt bei jüngeren Patienten sowie in der Schwangerschaft eingesetzt werden.

- Ein Screening auf Gerinnungsstörungen zur Prävention von erneuten venösen Thrombosen, zur Reduktion der Mortalität sowie zur Verbesserung des funktionellen Ergebnisses wird nicht generell empfohlen.

- Patienten mit Venen-/Sinusthrombose (CVST) sollen in der akuten Phase mit Heparin in einer therapeutischen Dosis behandelt werden, unabhängig davon, ob bereits eine intrakranielle Blutung vorliegt oder nicht. Dabei kann vorzugsweise niedermolekulares statt unfraktioniertes Heparin eingesetzt werden.

- Trotz der insgesamt geringen Evidenz wird die operative Dekompression bei Patienten mit CVST,
Läsionen des Parenchyms (Stauungsödem und/ oder Hämorrhagie) und drohender Einklemmung zur Verhinderung von Todesfällen empfohlen.

- Nach der Akutphase wird eine orale Antikoagulation mit einem Vitamin-K-Antagonisten für 3-12 Monate empfohlen zur Prävention einer RezidivCVST und extrazerebraler venöser Thrombosen.

- Frauen mit früherer CVST sollten auf die orale Einnahme kombinierter hormoneller Kontrazeptiva verzichten.

\section{Wissenschaftlich verantwortlich gemäß} Zertifizierungsbestimmungen

Wissenschaftlich verantwortlich gemäß

Zertifizierungsbestimmungen für diesen Beitrag ist Prof. Dr. med. Christian Weimar, Elzach.

\section{Zitierweise für diesen Artikel}

Fortschritte der Neurologie · Psychiatrie 2021; 89(4): 182-194 Dieser Beitrag ist eine aktualisierte Version des Artikels: Weimar C. Diagnostik und Therapie der zerebralen Venen-/ Sinusthrombose. Neurologie up2date 2019; 2(03): 267 - 279. DOI: 10.1055 /a-0829-9711

\section{Interessenkonflikt}

Erklärung zu finanziellen Interessen

Forschungsförderung erhalten: Ja, von einer anderen Institution (Pharma- oder Medizintechnikfirma usw.). Honorar/geldwerten Vorteil für Referententätigkeit erhalten: nein; Bezahlter Berater/interner Schulungsreferent/Gehaltsempfänger: nein; Patent/Geschäftsanteile/Aktien (Autor/Partner, Ehepartner, Kinder) an Firma (Nicht-Sponsor der Veranstaltung): ja; Patent/Geschäftsanteile/Aktien (Autor/Partner, Ehepartner, Kinder) an Firma (Sponsor der Veranstaltung): nein.

Erklärung zu nichtfinanziellen Interessen

Die Autorinnen/Autoren geben an, dass kein Interessenkonflikt besteht.

\section{Autorinnen / Autoren}

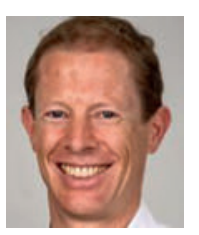

Prof. Dr. med. Christian Weimar war nach dem Medizinstudium mit Promotion in Freiburg seit 1996 an der neurologischen Universitätsklinik Essen tätig. Dort wurde er 2009 zum apl. Professor für Neurologie ernannt und ist seit 2020 Direktor der BDH Klinik Elzach. Seine wissenschaftlichen Tätigkeitsschwerpunkte beinhalten klinische Studien zu Prävention und Behandlung des Schlaganfalls sowie Epidemiologie und Bildgebung von Gedächtnisstörungen. 
Korrespondenzadresse

Prof. Dr. med. Christian Weimar

BDH-Klinik Elzach gGmbH

Ärztlicher Direktor

Am Tannwald 1

79215 Elzach

Deutschland

E-Mail: Christian.weimar@bdh-klinik-elzach.de

Literatur

[1] Coutinho JM, Zuurbier SM, Aramideh M et al. The incidence of cerebral venous thrombosis: a cross-sectional study. Stroke 2012; 43: 3375-3377

[2] Bousser MG, Crassard I. Cerebral venous thrombosis, pregnancy and oral contraceptives. Thromb Res 2012; 130 (Suppl 1): S19-22

[3] Gameiro J, Ferro JM, Canhao P et al. Prognosis of cerebral vein thrombosis presenting as isolated headache: early vs. late diagnosis. Cephalalgia 2012; 32: 407-412

[4] Ferro JM, Canhao P, Stam J et al. Prognosis of cerebral vein and dural sinus thrombosis: results of the International Study on Cerebral Vein and Dural Sinus Thrombosis (ISCVT). Stroke 2004; 35: 664-670

[5] Chik Y, Gottesman RF, Zeiler SR et al. Differentiation of transverse sinus thrombosis from congenitally atretic cerebral transverse sinus with CT. Stroke 2012; 43: 1968-1970

[6] Stam J, de Bruijn S, deVeber G. Anticoagulation for cerebral sinus thrombosis. Cochrane Database Syst Rev 2008; 8: CD002005

[7] Ferro JM, Bousser MG, Canhao P et al. European Stroke Organization guideline for the diagnosis and treatment of cerebral venous thrombosis - endorsed by the European Academy of Neurology. Eur J Neurol 2017; 24: 1203-1213. doi: 10.1111/ ene. 13381

[8] Arbeitsgemeinschaft Wissenschaftlich-Medizinischer Fachgesellschaften-AWMF. Diagnostik und Therapie der Venenthrombose und der Lungenembolie. Stand: 10.10.2015, gültig bis 09.10.2020. AWMF-Leitlinienregister 065/002. Im Internet: https://www.awmf.org/leitlinien/detail/II/065-002.html (Stand: 15. 01.2021)

[9] Lebas A, Chabrier S, Fluss J et al. EPNS/ SFNP guideline on the anticoagulant treatment of cerebral sinovenous thrombosis in children and neonates. Eur J Paediatr Neurol 2012; 16: 219228

[10] Coutinho JM, Zuurbier SM, Bousser MG, Ji X, Canhao P, Roos YB, et al. Effect of Endovascular Treatment With Medical Management vs Standard Care on Severe Cerebral Venous Thrombosis: The TO-ACT Randomized Clinical Trial. JAMA Neurol. 2020;8;77(8):966-973

[11] Dentali F, Squizzato A, Gianni M et al. Safety of thrombolysis in cerebral venous thrombosis. A systematic review of the literature. Thromb Haemost 2010; 104: 1055-1062
[12] Dentali F, Poli D, Scoditti U et al. Long-term outcomes of patients with cerebral vein thrombosis: a multicenter study. J Thromb Haemost 2012; 10: 1297-1302

[13] Ferro JM, Coutinho JM, Dentali F, Kobayashi A, Alasheev A, Canhao P, et al. Safety and Efficacy of Dabigatran Etexilate vs Dose-Adjusted Warfarin in Patients With Cerebral Venous Thrombosis: A Randomized Clinical Trial. JAMA Neurol. 2019; 3;76(12):1457-1465.

[14] Bousser MG, Ferro JM. Cerebral venous thrombosis: an update. Lancet Neurol 2007; 6: 162-170

[15] Bugnicourt JM, Guegan-Massardier E, Roussel M et al. Cognitive impairment after cerebral venous thrombosis: a two-center study. J Neurol 2013; 260: 1324-1331

[16] Saposnik G, Barinagarrementeria F, Brown RD Jr et al. Diagnosis and management of cerebral venous thrombosis: a statement for healthcare professionals from the American Heart Association/American Stroke Association. Stroke 2011; 42: 1158-1192

[17] Masuhr F, Busch M, Amberger $\mathrm{N}$ et al. Risk and predictors of early epileptic seizures in acute cerebral venous and sinus thrombosis. Eur ] Neurol 2006; 13: 852-856

[18] Ferro JM, Canhao P, Bousser MG et al. Early seizures in cerebral vein and dural sinus thrombosis: risk factors and role of antiepileptics. Stroke 2008; 39: 1152-1158

[19] Ferro JM, Correia M, Rosas MJ et al. Seizures in cerebral vein and dural sinus thrombosis. Cerebrovasc Dis 2003; 15: 78-83

[20] Palazzo P, Agius P, Ingrand P et al. Venous thrombotic recurrence after cerebral venous thrombosis: a long-term followup study. Stroke 2017; 48: 321-326. doi: 10.1161/ STROKEAHA.116.015294

[21] Arbeitsgemeinschaft Wissenschaftlich-Medizinischer Fachgesellschaften - AWMF. Prophylaxe der venösen Thromboembolie (VTE). Stand: 15.10.2015, gültig bis 14.10.2020. AWMFLeitlinienregister 003/001. Im Internet: https://www.awmf.o rg/leitlinien/detail/II/003-001.html (Stand: 15.01.2021)

[22] Monagle P, Chalmers E, Chan A et al. Antithrombotic therapy in neonates and children. American College of Chest Physicians Evidence-Based Clinical Practice Guidelines, 8th edn. Chest 2008; 133: 887S-968S

[23] Aguiar de Sousa D, Canhao P, Ferro JM. Safety of pregnancy after cerebral venous thrombosis: a systematic review. Stroke 2016; 47: 713-718. doi: 10.1161/STROKEAHA.115.011955

[24] Bain E, Wilson A, Tooher R et al. Prophylaxis for venous thromboembolic disease in pregnancy and the early postnatal period. Cochrane Database Syst Rev 2014; 2: 2):CD001689. doi: 10.1002/14651858.CD001689

\section{Bibliografie}

Fortschr Neurol Psychiatr 2021; 89: 182-194

DOI 10.1055/a-1323-1563

ISSN 0720-4299

(C) 2021. Thieme. All rights reserved.

Georg Thieme Verlag KG, Rüdigerstraße 14,

70469 Stuttgart, Germany 


\section{Punkte sammeln auf CMIE.thieme.de}

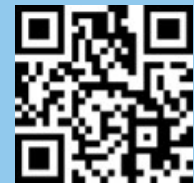

Diese Fortbildungseinheit ist in der Regel 12 Monate online für die Teilnahme verfügbar.

Den genauen Einsendeschluss finden Sie unter https://eref.thieme.de/CXG6P1Q.

Sollten Sie Fragen zu Online-Teilnahme haben, finden Sie unter https://cme.thieme.de/hilfe eine ausführliche Anleitung. Wir wünschen viel Erfolg beim Beantworten der Fragen!

Unter https://eref.thieme.de/CXG6P1Q oder über den QR-Code kommen Sie direkt zur Startseite des Wissenstests.

VNR 2760512021160210534

\section{Frage 1}

In welcher der genannten Bevölkerungsgruppen ist die Wahrscheinlichkeit einer zerebralen Venen-/Sinusthrombose (CVST) am größten?

\section{A Frauen im Klimakterium \\ B Frauen mit Herzmuskelhypertrophie \\ C Männer mit Herzmuskelhypertrophie \\ D Frauen post partum \\ E Kindesalter}

\section{Frage 2}

Welches ist kein Risikofaktor für einer zerebralen Venen-/ Sinusthrombosen?
A Einnahme von Steroiden
B Enzephalitis
C Hepatitis
D Leberzirrhose
E Magenatonie

\section{Frage 3}

Welche Aussage zur Pathophysiologie der CVST trifft zu?
A Eine chronische Sinusitis führt oft zu einer Thrombose des Sinus cavernosus.
B Eine Hirndruckerhöhung bei CVST ist direkt bedingt durch die venöse Abflussstauung in einem zerebralen Sinus.
C Hypoplastische Sinus stellen eine Prädisposition für CVST dar.
D Eine Liquorresorptionsstörung spielt für die Hirndrucker- höhung bei CVST keine Rolle.
E Eine Stauungsblutung bei CVST deutet auf eine venöse Stase in kortikalen Venen hin.

\section{Frage 4}

Welcher der genannten Befunde ist das häufigste Erstsymptom einer zerebralen Venen-/Sinusthrombose?

$\begin{array}{ll}\text { A } & \text { Bewusstseinsstörungen } \\ \text { B } & \text { epileptischer Anfall } \\ \text { C } & \text { Kopfschmerzen } \\ \text { D } & \text { Tinnitus } \\ \text { E } & \text { Übelkeit/Erbrechen }\end{array}$

\section{Frage 5}

Welche Aussage zur Bildgebung bei CVST trifft zu?
A Eine MR mit Angiografie ist der CCT mit Angiografie im Nachweis von kortikalen Thrombosen sowie Thrombosen tiefer Hirnvenen überlegen.
B Eine MRT mit Angiografie sollte immer der CCT mit Angio- grafie vorgezogen werden.
C Eine venöse Thrombose kann mittels MRT auch ohne Gefäß- darstellung ausgeschlossen werden.
D Eine digitale Angiografie als Goldstandard sollte in Zweifels- fällen immer erfolgen.
E Mittels MR oder CT Angiografie lässt sich eine Hypoplasie eines Sinus sicher von einer Thrombose unterscheiden.

\section{Frage 6}

Wodurch ist die Akuttherapie einer CVST gekennzeichnet?

A Eine PTT-wirksame (PTT = partielle Thromboplastinzeit) Heparinisierung ist erst nach Blutungsausschluss bzw. -resorption sinnvoll.

B NMWH (niedermolekulares Heparin) sollte in der Akutphase in der üblichen thromboseprophylaktischen Dosierung gegeben werden.

C Unfraktioniertes Heparin ist niedermolekularem Heparin in der Akutphase aufgrund der besseren Steuerbarkeit vorzuziehen.

D Direkte orale Antikoagulanzien sind eine evidenzbasierte Alternative zu Heparin.

E NMWH hat in der Akutphase der Thrombosebehandlung ein geringeres Blutungsrisiko als unfraktioniertes Heparin. 


\section{Punkte sammeln auf CMI:thieme.de}

Fortsetzung $\ldots$

\section{Frage 7}

Worauf deutet die Befundkombination aus Kopfschmerzen, motorischen Ausfällen, epileptischen Anfällen, Papillenödem und Bewusstseinsstörung hin?
A Thrombose im Sinus rectus
B Thrombose im Sinus transversus
C Thrombose im Sinus sigmoideus
D Thrombose im Sinus sagittalis superior
E Hirnödem der Basalganglien

\section{Frage 8}

In welcher Risikosituation wird nach Absetzen einer oralen Antikoagulation keine erneute Thromboseprophylaxe empfohlen?
A bei längeren Flugreisen
B bei immobilisierenden Erkrankungen
C bei erneuter Einnahme von Kontrazeptiva
D bei einer erneuten Schwangerschaft und danach im Wochenbett

E Kinder und Jugendliche bei erneuter Exposition von E. coli, Asparaginase und Steroiden sowie bei Anlage zentraler Venenkatheter

\section{Frage 9}

Nur eine der folgenden Aussagen zur Prognose nach CVST trifft zu. Welche?

A Das Risiko für ein erneutes thromboembolisches Ereignis ist nach Ablauf von 2 Jahren nicht mehr erhöht.

B Eine Rekanalisierung des thrombosierten Sinus ist der beste Prädiktor für Beschwerdefreiheit nach CVST.

C Mehr als die Hälfte aller Patienten leiden nach CVST an Kopfschmerzen, Anpassungs- oder Angststörungen.

D Nach epileptischen Anfällen während der Akutphase besteht ein hohes Risiko für die Ausbildung einer strukturellen Epilepsie.

E Die prophylaktische Gabe von Antiepileptika senkt das Risiko für eine spätere Epilepsie.

\section{Frage 10}

Wie groß ist der Anteil der Patienten mit zerebraler Venen-/ Sinusthrombose, bei denen in der Bildgebung ein Hirnödem nachweisbar ist?
A ca. $5 \%$
B ca. $20 \%$
C ca. $33 \%$
D ca. $50 \%$
E ca. $75 \%$ 